\title{
Yeast proteins do not practice social distancing as species hybridize
}

\author{
Caroline M. Berger ${ }^{1,2,3,4}$ (D) Christian R. Landry ${ }^{1,2,3,4}$
}

Received: 15 January 2021 / Accepted: 12 April 2021 / Published online: 4 May 2021

(c) The Author(s), under exclusive licence to Springer-Verlag GmbH Germany, part of Springer Nature 2021

\begin{abstract}
With the current COVID-19 pandemic, we all realized how important interactions are. Interactions are everywhere. At the cellular level, protein interactions play a key role and their ensemble, also called interactome, is often referred as the basic building blocks of life. Given its importance, the maintenance of the integrity of the interactome is a real challenge in the cell. Many events during evolution can disrupt interactomes and potentially result in different characteristics for the organisms. However, the molecular underpinnings of changes in interactions at the cellular level are still largely unexplored. Among the perturbations, hybridization puts in contact two different interactomes, which may lead to many changes in the protein interaction network of the hybrid, including gains and losses of interactions. We recently investigated the fate of the interactomes after hybridization between yeast species using a comparative proteomics approach. A large-scale conservation of the interactions was observed in hybrids, but we also noticed the presence of proteostasis-related changes. This suggests that, despite a general robustness, small differences may accumulate in hybrids and perturb their protein physiology. Here, we summarize our work with a broader perspective on the importance of interactions.
\end{abstract}

Keywords Protein-protein interaction $\cdot$ Interactome $\cdot$ Hybridization $\cdot$ Yeast

\section{Interactions happen everywhere}

Understanding the mechanisms that underlie the multiplicity of traits, or phenotypic diversity, presents a substantial challenge in evolutionary biology. Network science could be one step towards a better comprehension. The current pandemic has made networks part of our everyday lives. However, such networks have always been everywhere around us. In space, stars interact with their planets through gravitation, radiation and magnetic fields (Vidotto 2019). In nature,

Communicated by Michael Polymenis.

Caroline M. Berger

caroline.berger.1@ulaval.ca

1 Département de Biologie, Faculté des sciences et de génie, Université Laval, Quebec, QC G1V0A6, Canada

2 Département de Biochimie, Microbiologie et Bio-informatique, Faculté des sciences et de génie, Université Laval, Quebec, QC G1V0A6, Canada

3 Le réseau québécois de recherche sur la fonction, la structure et l'ingénierie de protéines, PROTEO, Université Laval, Quebec, QC G1V0A6, Canada

4 Centre de Recherche en Données Massives (CRDM), Université Laval, Quebec, QC G1V0A6, Canada interactions among species are hundreds. They range from short-term interactions (such as pollination and predation) to long-term interactions (such as mutualism that is beneficial for both partners, or competition that is harmful for the two) (Wootton and Emmerson 2005). Interactions exist also at a much lower level. Among the interactions that take place in the cell, protein interactions play a key role and can be seen as the basic building blocks of life. Proteins are known to interact with each other to form a complicated network called interactome, which includes protein complexes. The interactome has a significant role in many processes such as signal transduction, cell proliferation, growth, differentiation, and apoptosis (Diss et al. 2013). As a consequence, any changes in the interactome may have key impacts on the characteristics and phenotypes of organisms. Better apprehension of the events that can disrupt the interactome will provide a unique opportunity to investigate the molecular bases of phenotypic diversity. 


\section{Protein interactions can be disrupted}

Maintenance of the integrity of the interactome is a real challenge in the cell. Aberrant protein interactions have been associated with many human diseases such as cancer, infectious diseases and neurodegenerative diseases (Lu et al. 2020). The challenge is all the more important as many events can disrupt the interactome. Among them, mutations in genes can modify the interactome, and induce either fragility (Zhong et al. 2009) or robustness (Diss et al. 2017). Besides mutations, interactions between species are a major source of disruption for the interactome (Fig. 1). Host-pathogen interactions are a great example of interactions between species that disrupt the interactome (Mukhtar et al. 2011, Orit et al. 2012). The long coevolution between pathogens and their host should favor pathogens that successfully manipulate their host by targeting key proteins in their interaction network (Fig. 1a, b). Another interesting case of disruption occurs when two different species reproduce with each other: this is what we call hybridization. In hybrids, one can expect that we will observe a mix of the two parental interactions (Fig. 1c). The interactome will be composed of both intra-species (or parental) interactions and inter-species (or chimeric) interactions. Disruption of the interactome in the hybrid can result in either loss of interactions between proteins that normally interact within the parental species, or the formation of new hybrid-specific interactions.

\section{The fate of the interactome in hybrids}

The question of what is the fate of the interactome in hybrids is all the more interesting as hybrids can show very different phenotypes from their parents. The first idea that comes in mind is that hybrids will have intermediate characteristics from their parents. However, many studies have demonstrated the contrary. Sometimes, hybrids are "less efficient" than their parents, what we call underdominance (Maheshwari and Barbash 2011). For example, hybrids between indica and japonica subspecies of Asian cultivated rice are sterile (Ouyang et al. 2010). One the contrary, hybrids may be superior to their parents, a case that is called heterosis (Lippman and Zamir 2007). For example, hybrids between the common pheasant (Phasianus colchicus) and the red junglefowl (Gallus gallus) are bigger than their parents (Darwin 1868). We can easily imagine the economical importance of such hybrid species. One striking example is the hybrid corn that offers a considerable advantage in seed yield over parental species (Smith et al. 2004).

\section{a) Viral} infection
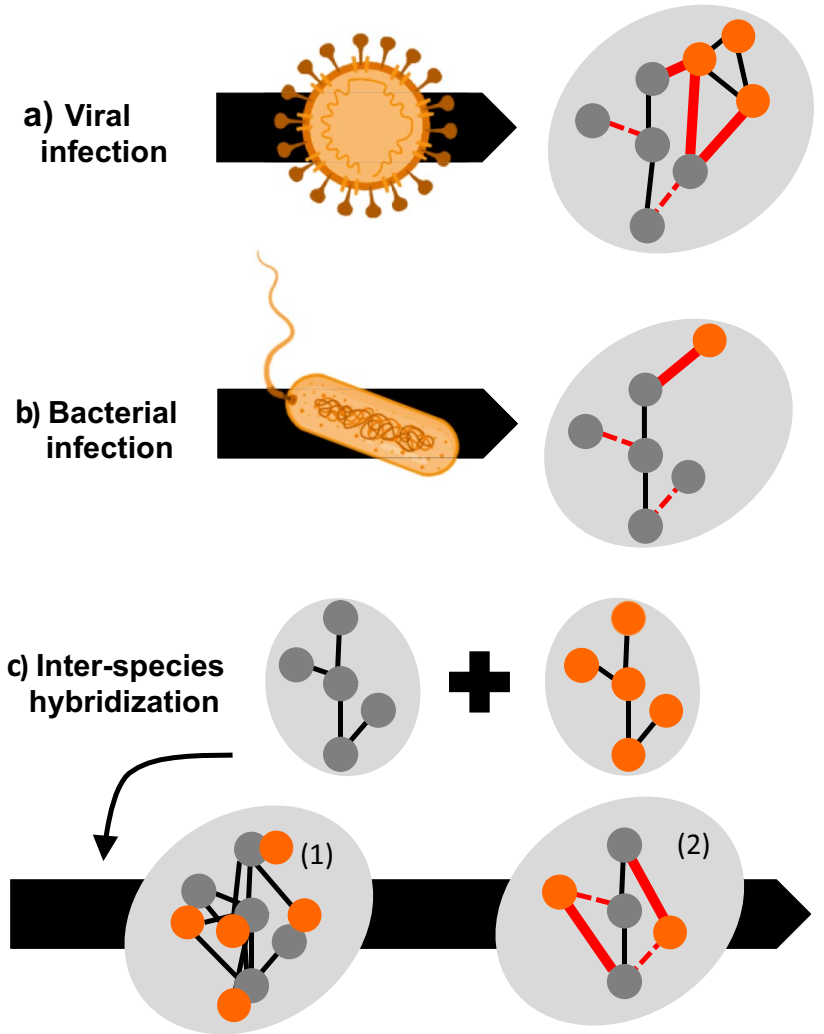

Fig. 1 Interactions between species can disrupt the interactome. a Viral infections (top panel) lead to contact between proteins of the host and the virus. Interactions between viral and host proteins (thick red lines) will disrupt the host interactome (red dotted lines) and therefore cellular processes. b In bacterial infections (center), the host interactome is disrupted by effector proteins from the bacteria (rather than by the entire interactome of the bacteria) (thick red line). These effectors can also modify the interactome of the host (red dotted lines). $\mathbf{c}$ In the case of hybridization between two distinct species (bottom panel), there is an initial mix of the two parental interactomes (step 1). After hybridization (step 2), the interactome of the hybrid will be composed of inter-species interactions also called chimeric interactions (red lines) and intra-species interactions also called parental interactions (black lines). Some interactions will have been disrupted (red dotted lines), while others will appear (thick red lines). Adapted from (Diss et al. 2013)

In all these cases, can protein interactome play a role in shaping these hybrid-specific characteristics? The answer is not clear, as previous studies tend to show different results. Some studies suggested the loss of interactions in hybrids because of a strong co-evolution, and thus incompatibility, between parental proteins (Lyad et al. 2012). On the contrary, some studies demonstrated that new chimeric interactions can appear in hybrids, and that this can be linked to enhanced functions in the cell (Piatkowska et al. 2013). However, these studies have some limitations. For instance, they focused on specific protein interactions and or specific protein complexes in the cell. Whether 
hybridization is associated with a global reorganization of the interactome is, therefore, mostly still unexplored.

\section{Comparative proteomics to study a large number of protein complexes}

In our recent paper, we used a method that combines sizeexclusion chromatography (SEC), protein correlation profiling (PCP) and stable isotope labeling by amino acids in cell culture (SILAC) to capture a large number of protein complexes in hybrids (Dandage et al. 2020). This method allows separating complex mixtures of endogenous proteins into a set of fractions, which are analyzed by mass spectrometry. Profiles of co-migrating proteins are clustered to reconstruct protein-protein interactions and, thus, protein complexes (Kristensen et al. 2012). In our study, SEC-PCP-SILAC was applied to the budding yeast Saccharomyces cerevisiae (parent 1) and to Saccharomyces uvarum (parent 2) - a closely related species belonging to the same genus (Scannell et al. 2011) and their hybrids (Fig. 2). Using yeast as a model is of special interest because spontaneous hybridization is common among yeast species and may have an important impact on their performance. During industrial processes such as wine making, hybridization is thought to be an important mechanism for adaptation to the environment, enhancing genetic flexibility and promoting adaptive change (González et al. 2006, Querol and Guillamon 2013). For example, hybrids between S. cerevisiae and Saccharomyces eubayanus acquire cryotolerance from $S$. eubayanus and beneficial fermentation from $S$. cerevisiae. Thus, the hybrids benefit from both ancestors, with the ability to utilize sugar and perform fermentation at low temperature, a condition frequently used in wine and beer making (Hebly et al. 2015, Krogerus et al. 2017). Considering the industrial applications, comprehensive understanding of the molecular underpinnings of hybrid characteristics is necessary.

\section{Robustness and changes in hybrids}

In our study, we investigated the assembly of a few dozen protein complexes in an untargeted manner. Most of the protein-protein interactions were similar between hybrids and their parents, and were consistent with the assembly of inter-species interactions (chimeric complexes) in hybrids (Fig. 2b). One of the complex that appears to be chimeric in hybrids was the prefoldin complex. We decided to use this hexameric protein complex, involved in cytoplasmic folding of actin and tubulin monomers during cytoskeleton assembly (Millán-Zambrano and Chávez 2014), to validate the presence of chimeric complexes in hybrids. Using the dihydrofolate reductase (DHFR) protein-fragment complementation
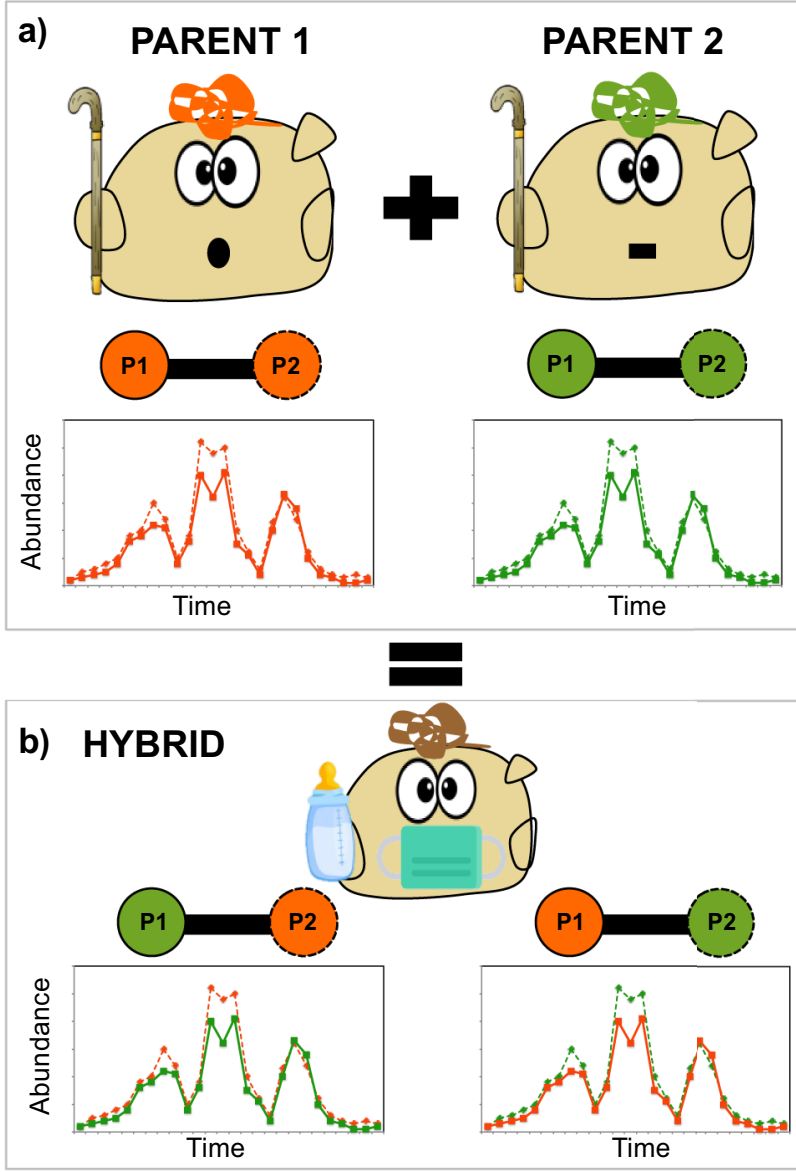

Fig. 2 SEC-PCP-SILAC enables comparing the interactomes of a parental and $\mathbf{b}$ hybrid yeast species. This method allows obtaining protein elution profiles that are subsequently used to infer protein interactions and protein complexes. The principle of the analysis is that two proteins ( $\mathrm{P} 1$ and $\mathrm{P} 2$ ) that interact will tend to have similar elution profiles over time. a Interaction between P1 and P2 is detected in both parents. b In our study, we demonstrated that after hybridization, there is a mix of the two parental interactomes: P1 of parent two interacts with $\mathrm{P} 2$ of parent one and the reverse, $\mathrm{P} 1$ of parent one interacts with $\mathrm{P} 2$ of parent two. The interactome of the hybrid is, thus, mostly composed of inter-species interactions (chimeric interactions)

assay (PCA) (Tarassov et al. 2008) applied to the prefoldin complex, we could confirm that chimeric interactions occur in hybrids.

Despite the overall general robustness of the protein complexes, we also detected cases of altered protein interactions in hybrids. Altered biological functions included functions linked to proteostasis and metabolism. We hypothesize that this could be an effect of or a response to the misregulation of protein proteostasis in hybrids, as many molecular traits are disrupted after hybridization (Bar-Zvi et al. 2017). This alteration could also be a result of imbalance among protein subunits of complexes in hybrids or incompatibilities between subunits. For instance, if the interaction between 
the two subgenomes produces proteins with altered stoichiometry, protein proteostasis mechanisms could be activated to restore proper protein balance.

\section{Further perspectives}

Upon comparison of the protein complexes in parents and their hybrids, we were able to uncover patterns that indicate robustness and mix of complexes between parents and hybrids. Robustness of protein complexes after hybridization was previously reported for two complexes, the nuclear pore complex and the RNA polymerase II (Leducq et al. 2012). The novelty of our study lies in the elucidation of the fate of protein complexes after hybridization for several protein complexes simultaneously. Leveraging on the availability of a broader survey of complexes, our study was able to highlight interesting differences in hybrids. Among them, proteostasis-related changes were detected, with enriched interactions in hybrids related to this biological function. Interestingly, similar results were obtained in a previous study that charted the hybrid proteome of a viable cross between Drosophila melanogaster females and Drosophila simulans males (Bamberger et al. 2018). In their study, the authors demonstrated that developing hybrids have $20 \%$ novel proteins in addition to parental species, including high levels of proteostasis-related proteins (heat shock proteins, proteasome-associated proteins, and proteasomal subunits). Thus, in light of this study and our own results, we can hypothesize that, on one hand, protein complexes are in general robust to hybridization. Social distancing is definitely not a long-term solution, even for yeast proteins. On the other hand, there might be incompatibilities between proteins of the different parents. These incompatibilities, of weak effects but distributed among many proteins, might result in perturbation of physiology of the cell and, thus, increased proteostasis. In the future, we aim to confirm the pathways that were identified as different between hybrids and parents. One possible avenue would be to study the evolution of the interactomes in hybrids over a long period of time and see, for instance, if proteostasis-related differences dissipate as hybrids evolve.

Acknowledgments We thank the members of the Landry Lab for discussions on the original manuscript.

Authors' contribution CMB wrote the paper with input from CRL.

Funding CMB was funded for the study reported in the paper by PROTEO via a FQRNT/PROTEO International Internship Program. This research was supported by the NSERC discovery grant to CRL and by Genome Canada and Genome British Columbia to Léonard J. Foster (Project 214PRO). CRL holds the Canada Research Chair in Canada Research Chair in Cellular Systems and Synthetic Biology. The mass spectrometry infrastructure used in the original paper was supported by the Canada Foundation for Innovation, the British Columbia Knowledge Development Fund and the BC Proteomics Network.

Availability of data and material Described in Dandage et al. (2020).

Code availability Described in Dandage et al. (2020).

\section{Declarations}

Conflicts of interest The authors declare that they have no conflict of interest.

\section{References}

Bamberger C, Martínez-Bartolomé S, Montgomery M, Lavallée-Adam M, Yates JR (2018) Increased proteomic complexity in Drosophila hybrids during development. Sci Adv. https://doi.org/10.1126/ sciadv.aao3424

Bar-Zvi D, Lupo O, Levy AA, Barkai N (2017) Hybrid vigor: the best of both parents or a genomic clash? Curr Opin Syst Biol 6:22-27. https://doi.org/10.1016/j.coisb.2017.08.004

Dandage R et al (2020) Frequent assembly of chimeric complexes in the protein interaction network of an interspecies yeast hybrid. Mol Biol Evol. https://doi.org/10.1093/molbev/msaa298

Darwin C (1868) The variation of animals and plants under domestication, Authorized. O. Judd \& Company, New York

Diss G, Filteau M, Freschi L, Leducq J-B, Rochette S, Torres-Quiroz F, Landry CR (2013) Integrative avenues for exploring the dynamics and evolution of protein interaction networks. Curr Opin Biotechnol 24:775-783. https://doi.org/10.1016/j.copbio.2013.02.023

Diss G, Gagnon-Arsenault I, Dion-Coté A-M, Vignaud H, Ascencio DI, Berger CM, Landry CR (2017) Gene duplication can impart fragility, not robustness, in the yeast protein interaction network. Science. https://doi.org/10.1126/science.aai7685

González SS, Barrio E, Gafner J, Querol A (2006) Natural hybrids from Saccharomycescerevisiae, Saccharomyces bayanus and Saccharomyces kudriavzevii in wine fermentations. FEMS Yeast Res 6:1221-1234. https://doi.org/10.1111/j.1567-1364.2006.00126.x

Hebly M, Brickwedde A, Bolat I, Nielsen J (2015) S. cerevisiae $\times$ $S$. eubayanus interspecific hybrid, the best of both worlds and beyond. FEMS Yeast Res. https://doi.org/10.1093/femsyr/fov005

Kristensen AR, Joerg G, Leonard JF (2012) A high-throughput approach for measuring temporal changes in the interactome. Nat Methods 9:907. https://doi.org/10.1038/nmeth.2131

Krogerus K, Magalhães F, Vidgren V, Gibson B (2017) Novel brewing yeast hybrids: creation and application. Appl Microbiol Biotechnol 101:65-78. https://doi.org/10.1007/s00253-016-8007-5

Leducq J-B, Charron G, Diss G, Gagnon-Arsenault I, Dubé AK, Landry CR (2012) Evidence for the robustness of protein complexes to inter species hybridization protein complexes in hybrids protein complexes in hybrids. PLoS Genet. https://doi.org/10.1371/journ al.pgen.1003161

Lippman ZB, Zamir D (2007) Heterosis: revisiting the magic. Trends Genet. https://doi.org/10.1016/j.tig.2006.12.006

Lu H, Zhou Q, He J, Jiang Z, Peng C, Tong R, Shi J (2020) Recent advances in the development of protein-protein interactions modulators: mechanisms and clinical trials. Signal Transduct Targ Ther. https://doi.org/10.1038/s41392-020-00315-3

Lyad Z, Marianna Z, Yearit F, Hadas N-G, Eitan R, Amir A (2012) Tight coevolution of proliferating cell nuclear antigen PCNA partner interaction networks in fungi leads to interspecies network 
incompatibility. Proc Natl Acad Sci. https://doi.org/10.1073/pnas. 1108633109

Maheshwari S, Barbash DA (2011) The genetics of hybrid incompatibilities. Annu Rev Genet 45:331-355. https://doi.org/10.1146/ annurev-genet-110410-132514

Millán-Zambrano G, Chávez S (2014) Nuclear functions of prefoldin. Open Biol. https://doi.org/10.1098/rsob.140085

Mukhtar MS et al (2011) Independently evolved virulence effectors converge onto hubs in a plant immune system network. Science. https://doi.org/10.1126/science.1203659

Orit R-R et al (2012) Interpreting cancer genomes using systematic host network perturbations by tumour virus proteins. Nature 487:491. https://doi.org/10.1038/nature11288

Ouyang Y, Liu Y-G, Zhang Q (2010) Hybrid sterility in plant: stories from rice. Curr Opin Plant Biol 13:186-192. https://doi.org/10. 1016/j.pbi.2010.01.002

Piatkowska EM, Naseeb S, Knight D, Delneri D (2013) Chimeric protein complexes in hybrid species generate novel phenotypes (proteome cross-talk impact on hybrid fitness) proteome cross-talk impact on hybrid fitness 9:e1003836. PLoS Genet. https://doi.org/ 10.1371/journal.pgen.1003836

Querol A, Guillamon JM (2013) Metabolomic comparison of Saccharomycescerevisiae and the Cryotolerant species $S$. bayanus var. uvarum and $S$. kudriavzevii during wine fermentation at low temperature (Research Article). PLoS One 8:e60135. https://doi. org/10.1371/journal.pone.0060135
Scannell DR, Zill OA, Rokas A, Payen C, Rine J, Johnston M, Hittinger CT (2011) The awesome power of yeast evolutionary genetics: new genome sequences and strain resources for the Saccharomyces sensu stricto genus. G3: Genes|Genomes|Genet. https://doi. org/10.1534/g3.111.000273

Smith CW, Betrán J, Runge ECA (2004) Corn: origin, history, technology, and production. John Wiley, Hoboken, N.J.

Tarassov K et al (2008) An in vivo map of the yeast protein interactome. Science. https://doi.org/10.1126/science.1153878

Vidotto AA (2019) Different types of star-planet interactions. Proc Int Astron Union 15:259-267. https://doi.org/10.1017/S174392131 9009979

Wootton JT, Emmerson M (2005) Measurement of interaction strength in nature. Annu Rev Ecol Evol Syst 36:419-444. https://doi.org/ 10.1146/annurev.ecolsys.36.091704.175535

Zhong Q et al (2009) Edgetic perturbation models of human inherited disorders. Mol Syst Biol. https://doi.org/10.1038/msb.2009.80

Publisher's Note Springer Nature remains neutral with regard to jurisdictional claims in published maps and institutional affiliations. 